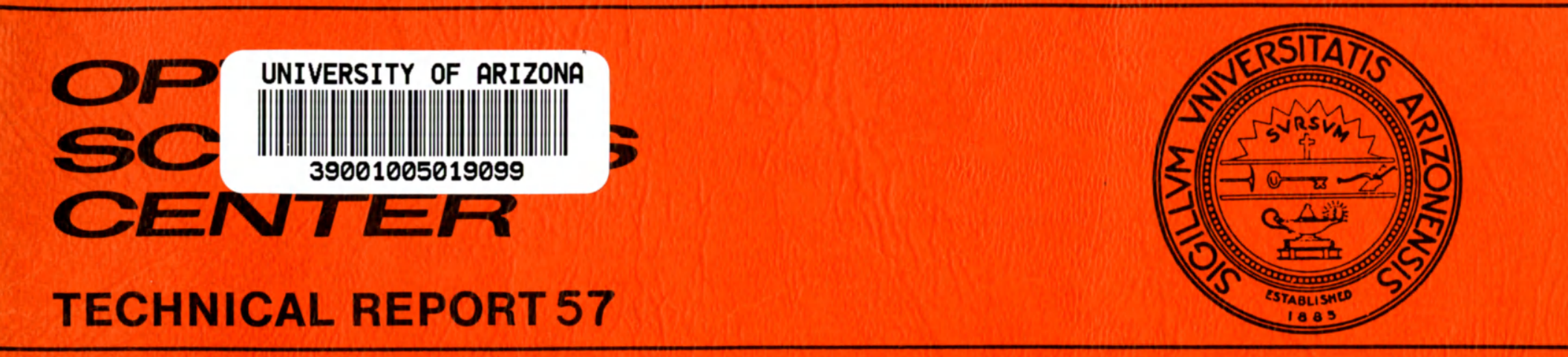

NORMALIZATION OF THE DELANO DIAGRAM

F. J. Lopez-López

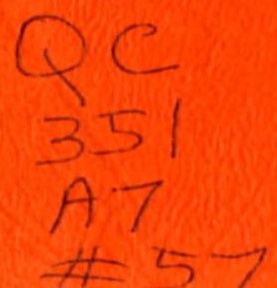




\title{
NORMALIZATION OF THE DELANO DIAGRAM
}

\author{
F. J. López-López
}

Optical Sciences Ceenter

The University of Arizona

Tucson, Arizona 85721

July 1970 


\section{FOREWORD}

This technical report is part of a dissertation being prepared in partial fulfillment of requirements for the PhD degree in Optical Sciences at the University of Arizona.

The text has been accepted for publication in Applied Optics. 


\begin{abstract}
A normalization of the Delano $y, \bar{y}$ diagram is proposed in which the $y$ heights are normalized by the entrance pupil height, the $\bar{y}$ heights by the image height. The normalization constants are expressed in terms of the system parameters, and it is seen that the reduced distances become normalized by the focal length of the system, the marginal ray reduced angles by the numerical aperture of the system, the chief ray angles by the field aperture, and the powers by the total power of the system. It is also shown that any number of refractions and transfers will not affect this normalization, but a stop or conjugate shift will destroy it and renormalization then becomes necessary.
\end{abstract}

DESCRIPTORS: First-order optics, Geometrical optics, Delano diagram, y, $\overline{\mathbf{y}}$ diagram 


\section{CONTENTS}

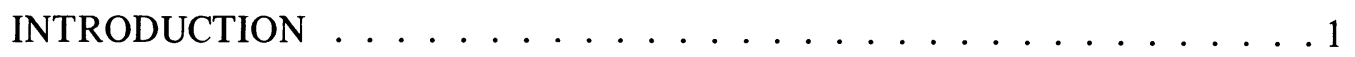

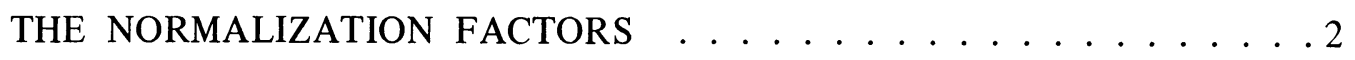

EFFECTS OF SHIFTS ON THE NORMALIZATION ........ 5

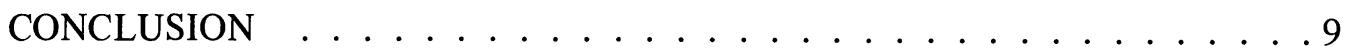

ACKNOWLEDGMENTS . . . . . . . . . . . . . 10 


\section{INTRODUCTION}

A useful tool in the analysis and design of optical systems in the paraxial or first order approximation is the $\mathrm{y}, \overline{\mathrm{y}}$ diagram, which was introduced by Delano in $1963 .{ }^{1}$ In many practical applications of this diagram it is convenient to work with normalized quantities of some sort. A normalized diagram permits a separation of specific properties from general ones. It is independent of the entrance pupil and image heights (which might be quite dissimilar, as in Cassegrain systems), producing a diagram that is easier to work with. It also facilitates the analytical treatment of the diagram. The results obtained by using a normalized diagram are applicable to a greater number of optical systems by simply changing the normalization factors. A normalized diagram also allows a fair comparison of several systems of widely different parameters.

This report presents a particular normalization that has been found very useful in practice. The normalization factors for separations, powers, and "reduced" angles are derived, and they are expressed in terms of such system parameters as focal length, field angle, and $f /$ number. In addition, the effects of refractions and transfers are investigated as well as of conjugate and stop shifts on an already normalized system.

In general we follow the same nomenclature as used by Delano, with the following exceptions: the indices of refraction are denoted by lower case $n$, the Lagrange invariant by the Russian letter $W($ (zhe), the object by subscript letter $O$, the image by subscript letter I, and the entrance and exit pupils by subscript letters $E$ and $E^{\prime}$, respectively. The figure below shows the symbols and quantities used.

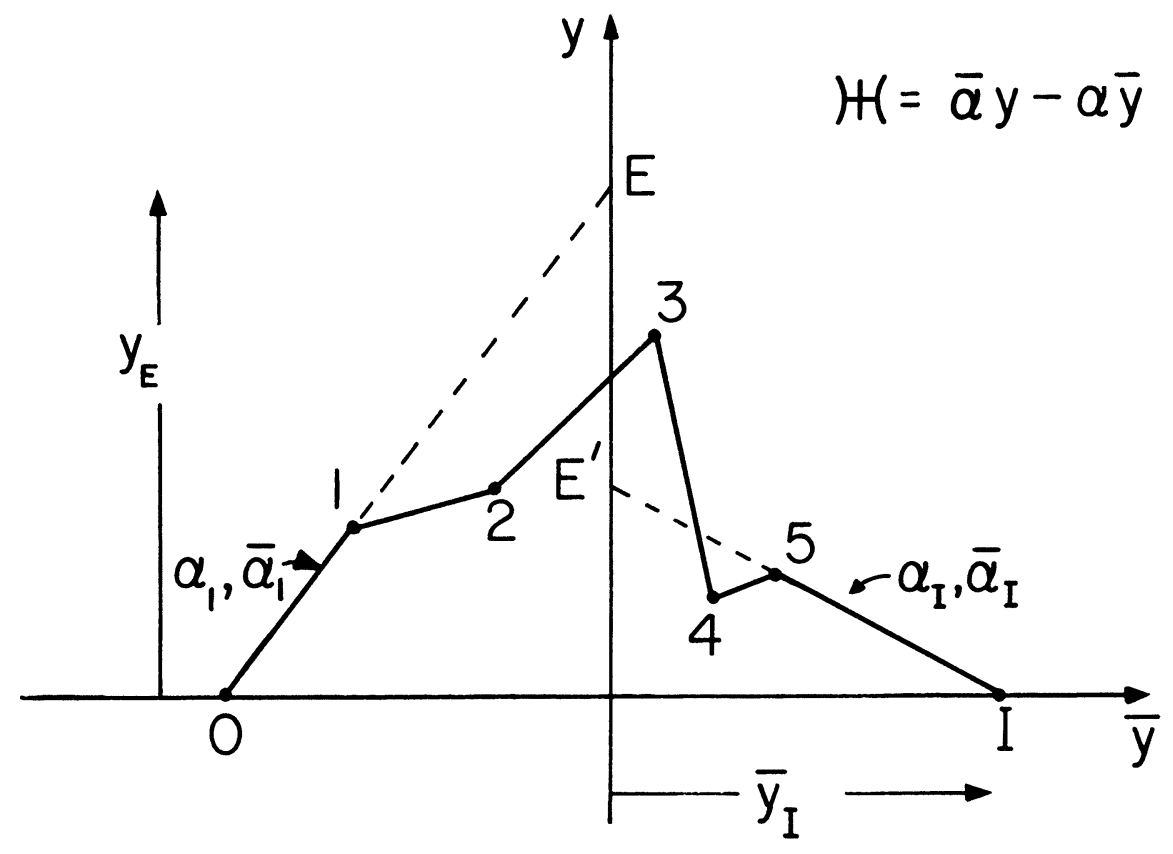

Fig. 1. Explanation of symbols and quantities used in the normalization. 


\section{THE NORMALIZATION FACTORS}

We shall use as normalization quantities the entrance pupil height and the height of the image.

Specifically, we divide all marginal ray heights by the entrance pupil height $\left(\mathrm{y}_{\mathrm{E}}\right)$, divide all the chief ray heights by the image height $\left(\bar{y}_{I}\right)$, and factor out the Lagrange invariant $(H)$ from all expressions.

As a result of this normalization, we shall see that the reduced distances are divided by a quantity proportional to the focal length of the system, the reduced angles for the marginal ray by the numerical aperture, the reduced angle for the chief ray by the field angle, and the powers of the components by the power of the system. In other words, the normalization is complete.

Using a circumflex $\left(^{\wedge}\right)$ over a letter to denote its normalized value, we may write:

$$
\left.\begin{array}{l}
\hat{y} \equiv y / y_{E}, \\
\hat{\bar{y}} \equiv \bar{y} / \bar{y}_{I},
\end{array}\right\}
$$

and by definition

$$
\hat{\mathrm{y}}_{\mathrm{E}}=\hat{\overline{\mathrm{y}}}_{\mathrm{I}}=\hat{\mathcal{W}}=1 \text {. }
$$

If we substitute the values (1) in the equations for the quantities derivable from the $y, \bar{y}$ diagram (Eqs. (1) in Delano 1963), we get the normalization factors as follows:

For the reduced distances

$$
\mathrm{D}_{\mathrm{i}}=\hat{\mathrm{D}}_{\mathrm{i}} \cdot \mathrm{y}_{\mathrm{E}} \overline{\mathrm{y}}_{\mathrm{I}} / \mathrm{W}
$$

For the reduced angles

$$
\begin{aligned}
& \alpha_{i}=\hat{\alpha}_{i} \cdot H / \bar{y}_{I} \\
& \bar{\alpha}_{i}=\widehat{\alpha}_{i} \cdot H / y_{E}
\end{aligned}
$$

And for the powers

$$
\mathrm{K}_{\mathrm{i}}=\hat{\mathrm{K}}_{\mathrm{i}} \cdot H /\left(\mathrm{y}_{\mathrm{E}} \overline{\mathrm{y}}_{\mathrm{I}}\right) \text {. }
$$

As in the article by Delano, the index $i$ refers to a surface or to the space preceding this surface.

It may also be noted that the Lagrange invariant does not change in form:

$$
W \equiv \bar{\alpha}_{i} y_{i}-\alpha_{i} \bar{y}_{i}=W\left(\hat{\bar{\alpha}}_{i} \hat{y}_{i}-\hat{\alpha}_{i} \hat{\bar{y}}_{i}\right)=W(\widehat{W} ;
$$

therefore

$$
\widehat{H} \equiv \hat{\bar{\alpha}}_{i} \hat{y}_{i}-\widehat{\alpha}_{i} \hat{\bar{y}}_{i}=1 \text {. }
$$


Although in many instances it is quite possible to specify the values of the normalizing quantities (the entrance pupil height and the height of the image) and the Lagrange invariant, often it is more convenient to express the normalization factors in terms of certain system parameters commonly used to specify a given optical system. Such parameters are the power (or focal length) of the system, its f-number, and the field angle. We shall use the following nomenclature:

$$
\begin{aligned}
& \mathrm{K}=\text { power of the system } \\
& \mathrm{f}^{\prime}=\mathrm{n}_{\mathrm{I}} / \mathrm{K}, \text { focal length } \\
& \mathrm{N}=\mathrm{f}-\text { number or f-ratio of system } \\
& \theta=2 \overline{\mathrm{u}}_{1}, \text { field angle } \\
& \hat{\mathrm{K}}=\text { correction factor derivable from the normalized diagram }
\end{aligned}
$$

We proceed to establish the values of the normalizing factors in terms of these system parameters.

From the expression of the power at each surface, we may see that the total power of the system is given by

where

$$
\mathrm{K}=\hat{\mathrm{K}} \cdot W /\left(\mathrm{y}_{\mathrm{E}} \overline{\mathrm{y}}_{\mathrm{I}}\right)
$$

$$
\hat{\mathrm{K}}=\widehat{\alpha}_{1} \hat{\bar{\alpha}}_{\mathrm{I}}-\widehat{\alpha}_{\mathrm{I}} \hat{\bar{\alpha}}_{1}=1+\widehat{\alpha}_{1} \hat{\bar{\alpha}}_{\mathrm{I}}
$$

because $\hat{\bar{\alpha}}_{1}=1, \hat{\alpha}_{I}=-1$, as may be seen from the value of the Lagrange invariant at the entrance pupil and at the image, respectively. We see also that this factor $\widehat{K}$, which acts as a correction factor, and whose value may be derived directly from the normalized diagram, will be unity when the object is located at infinity $\left(\hat{\alpha}_{1}=0\right)$, or the system is telecentric in image space $\left(\hat{\bar{\alpha}}_{I}=0\right)$.

Since $\mathrm{K}$ is given by Eq. (8), the normalization factor for powers is

$$
W / \mathrm{y}_{\mathrm{E}} \overline{\mathrm{y}}_{\mathrm{I}}=\mathrm{K} / \hat{\mathrm{K}} \text {, }
$$

that is, proportional to the power of the system; and the normalization factor for reduced distances is

$$
\mathrm{y}_{\mathrm{E}} \overline{\mathrm{y}}_{\mathrm{I}} / \mathrm{W}=\hat{\mathrm{K}} / \mathrm{K}=\hat{\mathrm{K}}\left(\mathrm{f}^{\prime} / \mathrm{n}_{\mathrm{I}}\right)
$$

that is, proportional to the focal length of the system.

From the value of the Lagrange invariant at the entrance pupil, we see that

$$
H / y_{E}=\bar{\alpha}_{1}=n_{1} \theta / 2,
$$

or the normalization factor for the chief ray angles is proportional to the field angle.

Similarly, from the value of the Lagrange invariant at the image, we have

$$
W / \bar{y}_{I}=-\alpha_{I},
$$

which is the numerical aperture of the system $\left(\alpha_{I} \equiv n_{I} u_{I}\right)$. This last normalization factor for the marginal ray angles may be expressed in terms of the f-number of the system. 
Let us define the f-number as

$$
\mathrm{N} \equiv \mathrm{f}^{\prime} /\left(2 \mathrm{y}_{\mathrm{E}}\right)=\mathrm{n}_{\mathrm{I}} /\left(2 \mathrm{~K} \mathrm{y}_{\mathrm{E}}\right)
$$

or equivalently

$$
\mathrm{y}_{\mathrm{E}}=\mathrm{n}_{\mathrm{I}} /(2 \mathrm{KN})
$$

From Eq. (10) we have, by using Eq. (15),

$$
W / \bar{y}_{I}=\left(n_{I} / \hat{K}\right)(1 / 2 N)
$$

which is another expression for the numerical aperture, valid for finite conjugates. The minus sign in Eq. (13) is on account of the sign convention for angles adopted here, which is the same as in analytic geometry.

Besides these normalization factors, the ratio $\bar{y}_{I} / y_{E}$ is useful in calculations of clear apertures and obstructions. Its value is easily derived from the above expressions:

$$
\overline{\mathrm{y}}_{\mathrm{I}} / \mathrm{y}_{\mathrm{E}}=\left(\mathrm{n}_{1} / \mathrm{n}_{\mathrm{I}}\right) \mathrm{N} \theta \widehat{\mathrm{K}}
$$

We may also express the Lagrange invariant as a function of the system parameters, namely

$$
W=\frac{n_{1} \theta f^{\prime}}{4 N}
$$




\section{EFFECTS OF SHIFTS ON THE NORMALIZATION}

We may see by direct substitution of Eq. (1) in the equations of paraxial ray tracing (Eq. (2) in Delano, 1963) that these equations retain their form after the normalization. In other words,

$$
\begin{array}{ll}
\text { Refraction: } & \hat{\alpha}_{\mathrm{i}+1}=\hat{\alpha}_{\mathrm{i}}-\hat{\mathrm{y}}_{\mathrm{i}} \hat{\mathrm{K}}_{\mathrm{i}} \\
\text { Transfer: } & \hat{\mathrm{y}}_{\mathrm{i}+1}=\hat{\mathrm{y}}_{\mathrm{i}}+\hat{\mathrm{D}}_{\mathrm{i}+1} \hat{\alpha}_{\mathrm{i}+1}
\end{array}
$$

and similar expressions for the chief ray. Therefore we conclude that the normalization of the diagram is not affected by any number of transfers and refractions that may occur between object and image points.

However, the normalization will be upset if we make either a stop shift (pupil shift) or a conjugate shift (object or image shift). A stop shift will change the scale of the marginal ray heights, defining a new entrance pupil height, and a conjugate shift will change the scale of the chief ray heights, defining a new image height.

A new normalization must be carried out with the new entrance pupil height or the new image height, according to which shift was made, in order to keep a normalized diagram. The normalization factors will be changed accordingly.

Let us see first the effect of the stop or pupil shift. As is explained in Delano's article, a stop shift is equivalent to a tilt of the y-axis, referring all points and lines to the resulting set of oblique axes. It can easily be shown that, after reorthogonalizing the axes, the net effect on points is to shift them parallel to the $\bar{y}$-axis, and the corresponding effect on the lines is to rotate them using their intersection with the $\bar{y}$-axis as a pivot. This is illustrated in Fig. 2 on the next page. We denote with a prime the new values after the stop shift.

The equations for a stop shift are given by

$$
\begin{array}{ll}
\hat{\overline{\mathrm{y}}}^{\prime}=\hat{\overline{\mathrm{y}}}-\hat{\mathrm{y}} / \mathrm{k}^{\prime} & \hat{\bar{\alpha}}^{\prime}=\hat{\bar{\alpha}}-\hat{\alpha} / \mathrm{k}^{\prime} \\
\hat{\mathrm{y}}^{\prime}=\hat{\mathrm{y}} & \hat{\alpha}^{\prime}=\hat{\alpha}
\end{array}
$$

where $\mathrm{k}^{\prime}$ is the slope of the new $\mathrm{y}^{\prime}$-axis. Accordingly, the new entrance pupil height is given by

$$
\hat{\mathrm{y}}_{\mathrm{E}}^{\prime}=1 \hat{\bar{\alpha}}_{1}^{\prime}=\mathrm{k}^{\prime} /\left(\mathrm{k}^{\prime}-\widehat{\alpha}_{1}\right)
$$

We see from here that, if $\mathrm{k}^{\prime}=\infty$, we have the trivial case of no stop shift. But if $\widehat{\alpha}_{1}=0$, that is, when the object is at infinity, although there is a shift of all points in the diagram, the line $\left(\widehat{\alpha}_{1}, \widehat{\bar{\alpha}}_{1}\right)$ does not change, and there is no change in the normalization.

For a conjugate shift, similar arguments apply: Tilting of the $\bar{y}$-axis and subsequent reorthogonalization of the axes leads to a shift of points parallel to the $y$-axis and rotation of the lines using as pivots their intersection with the y-axis. This is illustrated in Fig. 3. 


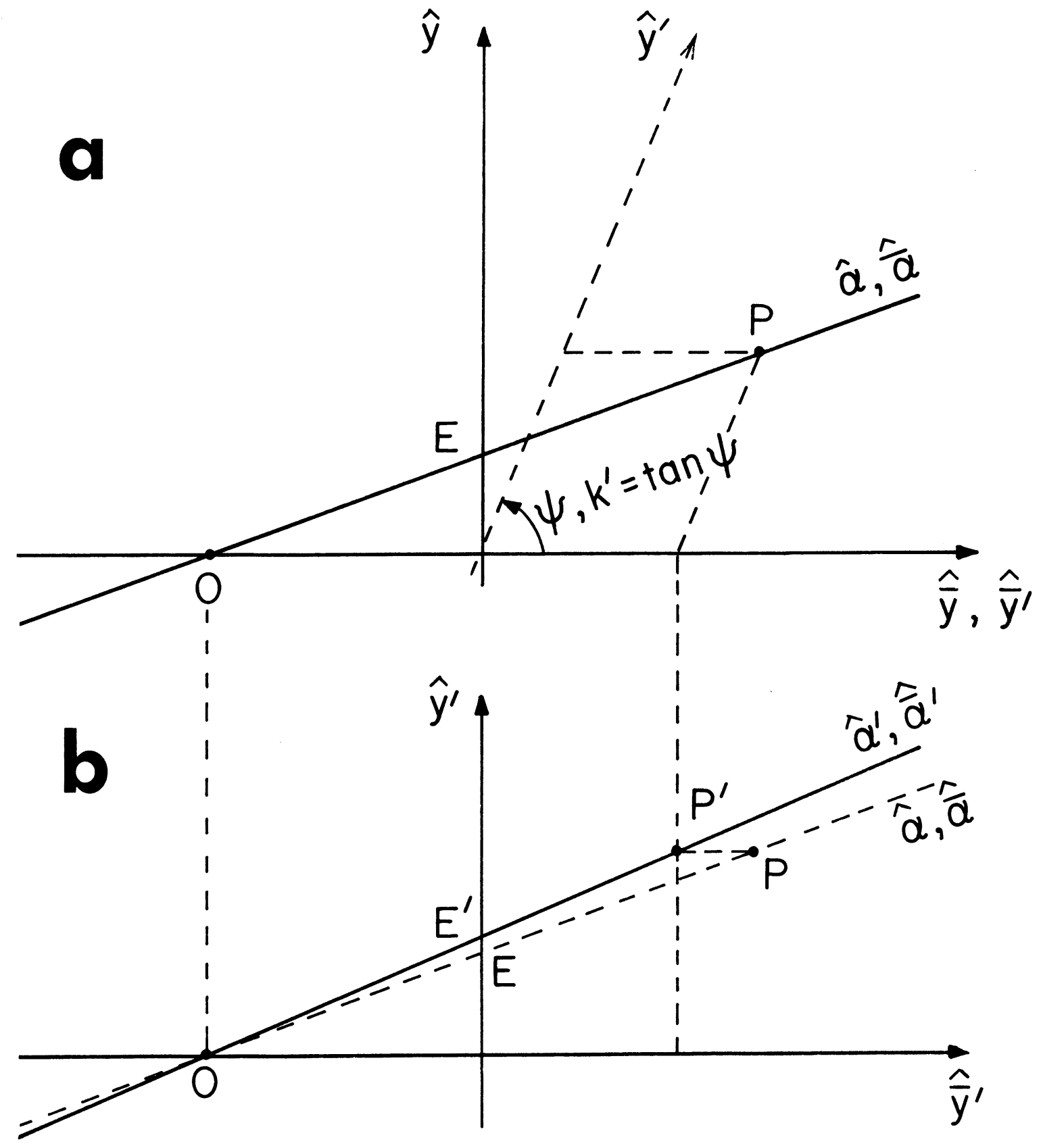

Fig. 2. a. Stop shift as a tilt of the $\hat{y}$-axis.

b. Net effect on a point $(P)$ and a line $(\hat{\alpha}, \widehat{\bar{\alpha}})$, after reorthogonalization of the axes. 

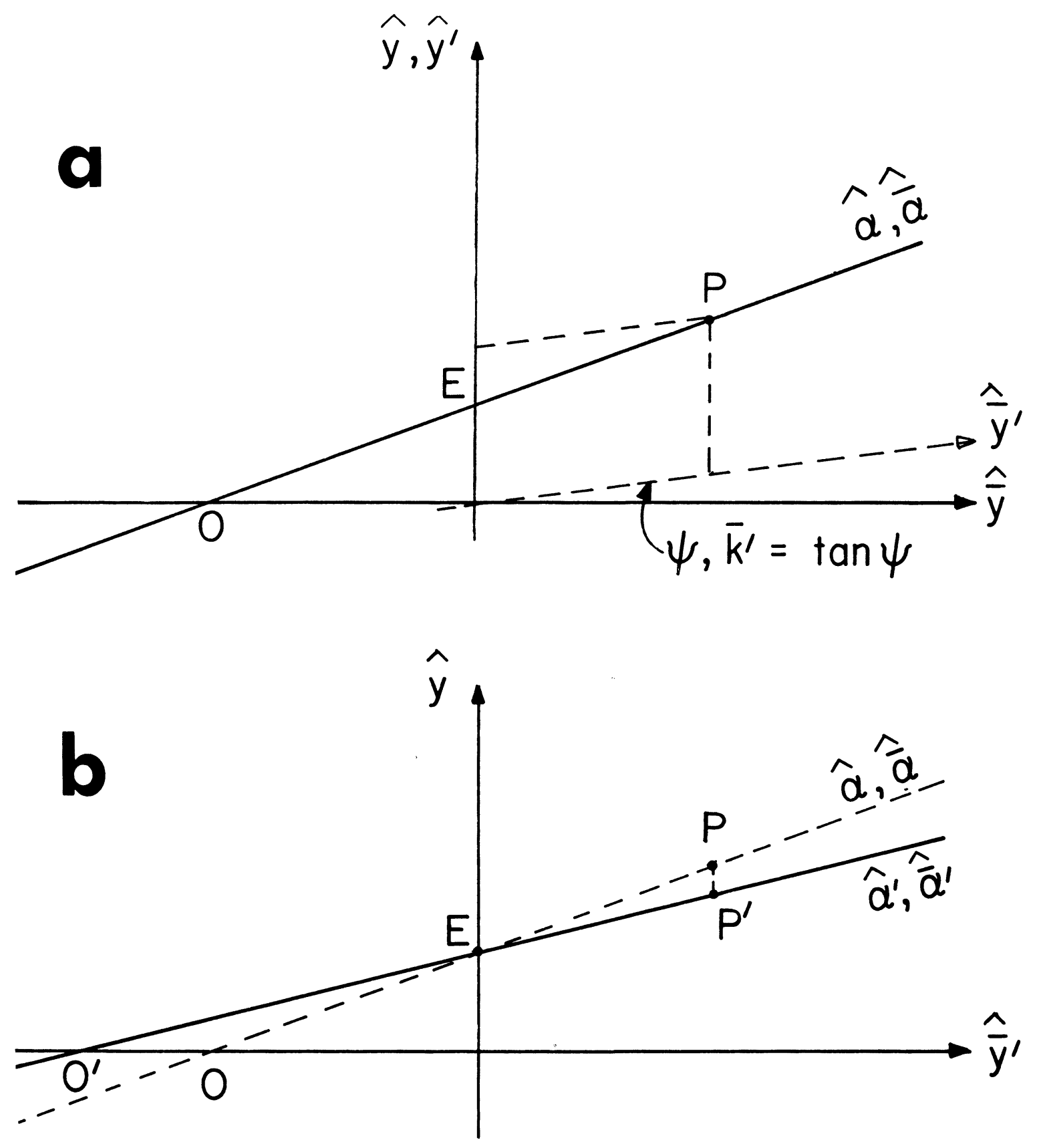

Fig. 3. a. Conjugate shift as a tilt of the $\hat{\bar{y}}$-axis.

b. Net effect on a point $(P)$ and a line $(\widehat{\alpha}, \hat{\bar{\alpha}})$, after reorthogonalization of the axes. 
The equations for the conjugate shift are

$$
\begin{array}{ll}
\hat{\bar{y}}^{\prime}=\hat{\bar{y}} & \hat{\bar{\alpha}}^{\prime}=\hat{\bar{\alpha}} \\
\hat{\mathrm{y}}^{\prime}=\hat{\mathrm{y}}-\mathrm{k}^{\prime} \hat{\overline{\mathrm{y}}} & \hat{\alpha}^{\prime}=\hat{\alpha}-\mathrm{k}^{\prime} \hat{\bar{\alpha}}
\end{array}
$$

where $\bar{k}^{\prime}$ is the slope of the new $\bar{y}^{\prime}$-axis. Then, the new height of the image is

$$
\hat{\bar{y}}_{I}^{\prime}=-1 / \widehat{\alpha}_{I}^{\prime}=1 /\left(\overline{\mathrm{k}}^{\prime} \hat{\bar{\alpha}}_{\mathrm{I}}+1\right) \text {. }
$$

We also see that, if $\bar{k}^{\prime}=0$, there will be no conjugate shift; but if $\hat{\bar{\alpha}}_{I}=0$, that is, if the system is telecentric in image space, then although there will be a shift given by Eq. (20), the value of $\hat{\alpha}_{I}$ will not change, and therefore the normalization is not affected.

As a result of these operations, either Eq. (19) or Eq. (21) will be different from unity-or perhaps both will be, if a stop shift and a conjugate shift are performed simultaneously. Therefore, we have to perform a new normalization using as normalizing quantities the values in Eqs. (19) and (21).

If we carry out this second normalization in the same way as was done before, Eqs. (1) through (6), we get new normalization factors that, expressed in terms of the original values, look like the following:

$$
\begin{array}{ll}
\text { For reduced distances and powers: } & \left(\mathrm{y}_{\mathrm{E}} \widehat{\mathrm{y}}_{\mathrm{E}}{ }^{\prime}\right)\left(\overline{\mathrm{y}}_{\mathrm{I}} \hat{\overline{\mathrm{y}}}_{\mathrm{I}}^{\prime}\right) / \mathbb{W} \\
\text { For reduced angles: } & \mathbb{H} /\left(\overline{\mathrm{y}}_{\mathrm{I}} \hat{\overline{\mathrm{y}}}_{\mathrm{I}}^{\prime}\right), \\
& \mathbb{W} /\left(\mathrm{y}_{\mathrm{E}} \hat{\mathrm{y}}_{\mathrm{E}}^{\prime}\right) .
\end{array}
$$

We see that the net effect of a shift and a renormalization is to change the normalizing quantities as follows:

$$
\begin{aligned}
& \mathrm{y}_{\mathrm{E}} \text { changes into } \mathrm{y}_{\mathrm{E}} \hat{\mathrm{y}}_{\mathrm{E}}{ }^{\prime} \\
& \overline{\mathrm{y}}_{\mathrm{I}} \text { changes into } \overline{\mathrm{y}}_{\mathrm{I}} \hat{\overline{\mathrm{y}}}_{\mathrm{I}}
\end{aligned}
$$

where $\mathrm{y}_{\mathrm{E}}, \overline{\mathrm{y}}_{\mathrm{I}}$ are the original normalizing quantities and $\hat{\mathrm{y}}_{\mathrm{E}}{ }^{\prime}, \hat{\overline{\mathrm{y}}}_{\mathrm{I}}^{\prime}$ are given by Eqs. (19) and (21).

We can conclude by induction that, if several stop shifts are performed in succession, the original entrance pupil has to be multiplied by corresponding factors to maintain a normalized diagram:

$$
\mathrm{y}_{\mathrm{E}} \rightarrow \mathrm{y}_{\mathrm{E}} \widehat{\mathrm{y}}_{\mathrm{E}}^{\prime} \widehat{\mathrm{y}}_{\mathrm{E}}^{\prime \prime} \widehat{\mathrm{y}}_{\mathrm{E}}^{\prime \prime \prime} \ldots
$$

and similarly for the conjugate shifts:

$$
\overline{\mathrm{y}}_{\mathrm{I}} \rightarrow \overline{\mathrm{y}}_{\mathrm{I}} \hat{\overline{\mathrm{y}}}_{\mathrm{I}}^{\prime \hat{\mathrm{y}}_{\mathrm{I}}} \hat{\mathrm{y}}_{\mathrm{I}}^{\prime \prime \prime} \ldots
$$

where each of the new factors has an expression equivalent to Eq. (19) or (21). 


\section{CONCLUSION}

We propose a normalization of the Delano $y-\bar{y}$ diagram such that all marginal ray heights are divided by the entrance pupil height and all the chief ray heights by the image height, and the Lagrange invariant is factored out.

The resulting normalization factors for the quantities derivable from the diagram can be expressed in terms of the system parameters (power $\mathrm{K}$, or focal length $\mathrm{f}$, f-number $\mathrm{N}$, and field angle $\theta$ ); these factors become

For reduced distances,

$$
\mathrm{D}_{\mathrm{i}} / \widehat{\mathrm{D}}_{\mathrm{i}}=\mathrm{y}_{\mathrm{E}} \overline{\mathrm{y}}_{\mathrm{I}} / \mathrm{H}=\left(\hat{\mathrm{K}} / \mathrm{n}_{\mathrm{I}}\right) \mathrm{f}^{\prime},
$$

which is seen to be proportional to the focal length of the system;

For chief ray reduced angles,

$\bar{\alpha}_{\mathrm{i}} / \hat{\bar{\alpha}}_{\mathrm{i}}=W / \mathrm{y}_{\mathrm{E}}=\bar{\alpha}_{1}=\mathrm{n}_{1} \theta / 2$,

which is proportional to the field angle;

For marginal ray reduced angles,

$\alpha_{\mathrm{i}} / \widehat{\alpha}_{\mathrm{i}}=H / \bar{y}_{\mathrm{I}}=-\alpha_{\mathrm{I}}=\left(\mathrm{n}_{\mathrm{I}} / \widehat{\mathrm{K}}\right)(1 / 2 \mathrm{~N})$,

which is proportional to the numerical aperture of the system;

For the powers,

$$
\mathrm{K}_{\mathrm{i}} / \widehat{\mathrm{K}}_{\mathrm{i}}=H / \mathrm{y}_{\mathrm{E}} \overline{\mathrm{y}}_{\mathrm{I}}=\mathrm{K} / \widehat{\mathrm{K}} \text {, }
$$

which is proportional to the power of the system.

The factor $\hat{\mathrm{K}}=1+\widehat{\alpha}_{1} \hat{\bar{\alpha}}_{\mathrm{I}}$ is derivable from the normalized diagram and will be equal to unity when the object is at infinity or when the system is telecentric in image space.

These normalization factors are unaffected by any number of refractions and transfers but have to be changed when a stop shift or a conjugate shift is performed in the already normalized diagram. Any shift and subsequent renormalization has the net effect of changing the original entrance pupil height or the height of the image, as the case may be, by

$$
\begin{aligned}
& \mathrm{y}_{\mathrm{E}} \rightarrow \mathrm{y}_{\mathrm{E}} \hat{\mathrm{y}}_{\mathrm{E}}^{\prime} \\
& \overline{\mathrm{y}}_{\mathrm{I}} \rightarrow \overline{\mathrm{y}}_{\mathrm{I}} \widehat{\hat{\mathrm{y}}}_{\mathrm{I}}^{\prime}
\end{aligned}
$$

where the expressions for $\hat{\mathrm{y}}_{\mathrm{E}}^{\prime}$ and $\hat{\overline{\mathrm{y}}}_{\mathrm{I}}^{\prime}$ are given by Eqs. (19) and (21). 


\section{ACKNOWLEDGMENTS}

I want to express my gratitude to Dr. Roland V. Shack for suggesting this problem and for his untiring patience and advice.

This work was supported in part by Project THEMIS, administered by the Air Force Office of Scientific Research. 


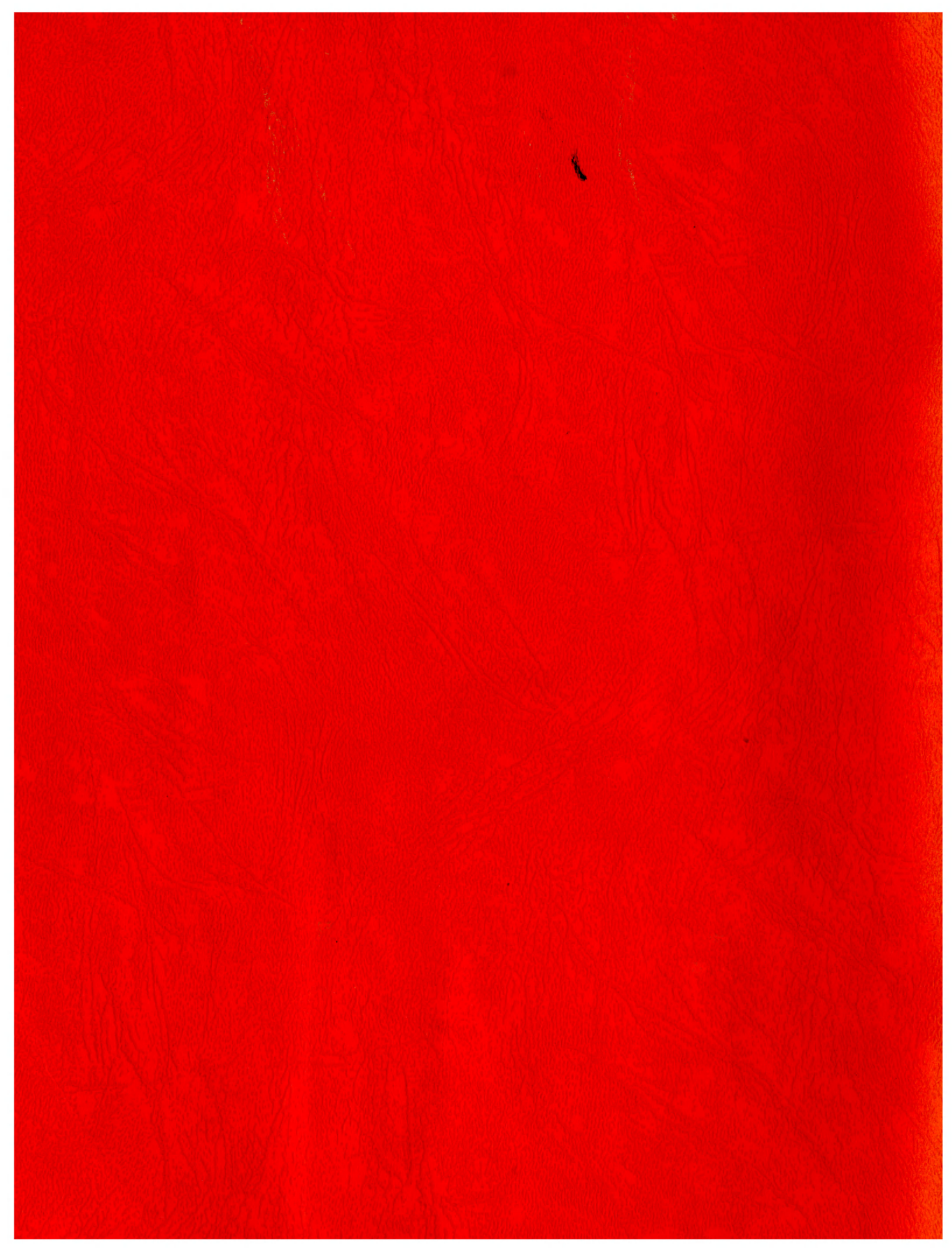

